\title{
Efeitos da suplementação de potássio via sal de cozinha sobre a pressão arterial e a resistência à insulina em pacientes obesos hipertensos em uso de diuréticos
}

\author{
Effects of potassium supplementation by salt on \\ arterial blood pressure and insulin resistance in hypertensive \\ obese patients on diuretic therapy
}

Maria Alice de Gouveia PEREIRA ${ }^{1}$

Roberto GALVÃO2

Maria Teresa ZANELLA ${ }^{3}$

\section{R E S U M O}

\section{Objetivo}

Avaliar os efeitos da suplementação de potássio, por intermédio do sal de cozinha contendo cloreto de potássio, associada à dieta hipocalórica e à atividade física aeróbica, sobre a pressão arterial e índices de resistência à insulina em pacientes hipertensos com obesidade abdominal.

\section{Métodos}

Estudo prospectivo duplo-cego, randomizado, em 22 pacientes hipertensos com excesso de peso (índice de massa corporal $\geq 27 \mathrm{~kg} / \mathrm{m}^{2}$ ) e controle insatisfatório da pressão arterial durante o uso de diuréticos pressão arterial sistólica $>140$ e $<160 \mathrm{mmHg}$ e/ou pressão arterial diastólica $>90$ e $<105 \mathrm{mmHg}$. O estudo teve duração de 12 semanas, durante as quais, os pacientes, divididos em dois grupos (grupo sal normal, $n=10$; grupo sal de potássio, $n=12$ ), receberam sal contendo $100 \%$ de cloreto de sódio, ou sal contendo $50 \%$ de cloreto de sódio e $50 \%$ de cloreto de potássio. No início e ao final do estudo, os pacientes foram submetidos à determinação do índice de massa corporal, da circunferência da cintura, dos níveis séricos e da excreção urinária de sódio e

\footnotetext{
1 Disciplinas de Nutrição e Metabolismo, Hospital do Rim e Hipertensão, Universidade Federal de São Paulo. Rua Borges Lagoa, 960, 04038-002, São Paulo, SP, Brasil. Correspondência para/Correspondence to: M.A.G. PEREIRA. E-mail: <alicegouveia@ig.com.br>.

2 Disciplina de Nefrologia, Hospital do Rim e Hipertensão, Universidade Federal de São Paulo. São Paulo, SP, Brasil.

3 Disciplina de Endocrinologia, Hospital do Rim e Hipertensão, Universidade Federal de São Paulo. São Paulo, SP, Brasil.
} 
potássio, à monitorização ambulatorial da pressão arterial nas 24 horas, ao teste oral de tolerância à glicose com determinação dos níveis séricos de insulina em jejum e aos 120 minutos, à determinação do perfil lipídico do plasma e à medida da composição corporal.

\section{Resultados}

No grupo sal de potássio, os níveis do potássio sérico não se elevaram, como no grupo sal normal, embora se elevasse de $38,8 \pm 18,6$ para $62,3 \pm 29,7 \mathrm{mEq} / \mathrm{g}$ a excreção de creatinina urinária $(p<0,05)$. A perda de peso, semelhante nos dois grupos (3,5\% no grupo sal normal e 2,7\% no grupo sal de potássio), associou-se às reduções na pressão arterial sistólica durante a monitorização ambulatorial da pressão arterial; de 134,7 $\pm 14,8$ para $130,2 \pm 12,6 \mathrm{mmHg}(p<0,05)$ no grupo sal normal e de $128,2 \pm 7,4$ para $122,9 \pm 5,7 \mathrm{mmHg}(p<0,05)$ no grupo sal de potássio, e às reduções na pressão arterial diastólica durante a monitorização ambulatorial da pressão arterial, de $84,4 \pm 10,2$ para $81,4 \pm 8,9 \mathrm{mmHg}$ no grupo sal normal e de $84,0 \pm 5,7$ para $79,5 \pm 3,9 \mathrm{mmHg}$ $(p<0,05)$ no grupo sal de potássio. As variações da pressão arterial sistólica, semelhantes nos dois grupos, correlacionaram-se com as variações das medidas da circunferência da cintura em todos os pacientes analisados em conjunto $\left(r_{s}=0,624 ; p=0,002\right)$. Os índices de resistência à insulina e o perfil lipídico do plasma não se alteraram e não diferiram entre os grupos.

\section{Conclusão}

Nossos resultados indicam que as perdas de peso induzidas por alterações no estilo de vida promovem reduções na pressão arterial, proporcionais às reduções na gordura abdominal. A suplementação de potássio a partir da utilização do sal de cozinha contendo cloreto de potássio, em pacientes hipertensos obesos em uso de diurético, se mostrou insuficiente para impedir a queda dos níveis séricos de potássio e não resultou em nenhum efeito sobre a resistência à insulina ou sobre a pressão arterial, além daquele obtido pela perda de peso.

Termos de indexação: cloreto de potássio, diuréticos, obesidade, pressão arterial, resistência à insulina.

\section{A B S T R A C T}

\section{Objective}

The objectives of the present study were to evaluate the effects of oral potassium supplementation, associated with a hypocaloric diet and aerobic exercises, on plasma potassium levels, blood pressure and insulin resistance, in centrally obese, not well controlled hypertensive patients on diuretic therapy; waist to hip ratio $>0.85$ in women, and $>0.95$ in men; systolic blood pressure $>140 \mathrm{mmHg}$ and $<160 \mathrm{mmHg}$ and/or diastolic blood pressure $>90 \mathrm{mmHg}$ and $<105 \mathrm{mmHg}$.

\section{Methods}

This was a prospective double-blind randomized study including 22 patients divided in 2 groups: sodium chloride $n=10$, and potassium chloride $n=12$. For 12 weeks, each group received cooking salt containing, either $100 \%$ sodium chloride, or $50 \%$ sodium chloride and $50 \%$ potassium chloride. All patients were submitted to a hypocaloric diet and advised to increase their physical activity; a 40-minute walk three times a week. Before and after the study period, all patients were submitted to determinations of body mass index, body composition, waist circumference, sodium and potassium urinary excretions, sodium and potassium serum levels, $24 \mathrm{~h}$ ambulatory blood pressure monitoring, oral glucose tolerance test with serum insulin measurements at fasting and 120 minutes after glucose load, and serum lipid profile.

\section{Results}

In both groups, no changes were observed in serum sodium and potassium levels, in blood glucose and insulin levels, insulin resistance indexes and serum lipid profile. The body mass index decreased similarly in 
both groups; $3.5 \pm 2.0 \%$ in sodium Chloride, and $2.7 \pm 3.2 \%$ in Potassium Chloride, as well as $24 \mathrm{~h}$ systolic ambulatory blood pressure monitoring mean; from $134.7 \pm 14.8$ to $130.2 \pm 12.6 \mathrm{mmHg}(p<0.05)$ in group sodium chloride, and from $128.2 \pm 7.4$ to $122.9 \pm 5.7 \mathrm{mmHg}(p<0.05)$ in potassium, and $24 \mathrm{~h}$ diastolic ambulatory blood pressure monitoring from $84.4 \pm 10.2$ to $81.4 \pm 8.9 \mathrm{mmHg}$ in sodium chloride, and from $84.0 \pm 5.7$ to $79.5 \pm 3.9 \mathrm{mmHg}(p<0.05)$ in group potassium $(p<0.05)$. Changes in $24 \mathrm{~h}$ systolic ambulatory blood pressure monitoring correlated with changes in the waist circumference, but not with changes in bory mass index when all patients were analyzed together. Serum lipid profile, blood glucose levels and insulin resistance indexes did not differ between groups and did not change during the study.

\section{Conclusion}

Weight reduction induced by life style changes, promotes reductions in blood pressure, which are proportional to reductions in abdominal fat. Potassium supplementation through cooking salt was insufficient to avoid serum potassium falling during diuretic therapy and did not show any additional beneficial effect on blood pressure or insulin resistance in hypertensive patients with central obesity.

Indexing terms: potassium chloride, diuretics, obesity, arterial blood pressure, insulin resistance.

\section{N T R O D U ÇÃ O}

A prevalência da hipertensão no Brasil pode assumir valores entre $11 \%$ e $20 \%{ }^{1}$, sendo o principal fator de risco para as doenças cardiovasculares. Para seu tratamento e prevenção, as estratégias não farmacológicas adotadas estão baseadas na modificação do estilo de vida, tendo como principais recomendações: a perda de peso, a redução da ingestão de sódio, a manutenção adequada da ingestão de potássio dietético e o aumento da atividade física².

Entre hipertensão e obesidade existe uma relação bem estabelecida: as pessoas obesas têm três vezes mais probabilidade de desenvolver hipertensão. O mecanismo possivelmente envolvido tem sido atribuído, em parte, à resistência à insulina e conseqüente hiperinsulinemia compensatória, mais freqüentemente presente em indivíduos com obesidade abdominal ou visceral ${ }^{3}$.

A resistência à insulina em pacientes hipertensos pode ser induzida pela hipocalemia que ocorre nos pacientes tratados com diuréticos thiazídicos, observando-se aumento nos níveis de insulina plasmática e intolerância à glicose associados ${ }^{4}$. O aumento nos níveis de insulina leva à maior absorção tubular de sódio. Este efeito tem sido implicado na fisiopatologia do estado hipertensivo em população de obesos ${ }^{5}$.

A perda de peso por meio de uma dieta hipocalórica, diminui a resistência à insulina, e a pressão sangüínea, reduzindo o risco cardiovascular ${ }^{6}$. No estudo TOHP II, em indivíduos com sobrepeso e pressão arterial elevada, perda de peso e redução da ingestão de sódio, sozinhos e combinados, foram efetivos para a diminuição da pressão sangüínea ${ }^{7}$. Mesmo quando a perda de peso é modesta, ocorre uma redução clinicamente significante na pressão arterial e diminui, portanto, o risco de hipertensão ${ }^{8}$. He et al. ${ }^{9}$, demonstraram que, depois de 7 anos, o efeito benéfico da perda de peso corporal no risco de desenvolvimento da hipertensão, ainda permanece.

Embora as dietas com conteúdos reduzidos de sal e agentes natriuréticos tenham sido associadas à redução da pressão sangüínea em pacientes hipertensos ${ }^{10}$, uma redução severa na ingestão de sódio não tem sido recomendada. Graudal et al. ${ }^{11}$, em seu estudo de meta-análise sugeriram que a magnitude do efeito da redução na ingestão de sódio na pressão sangüínea, embora consistente, não justifica uma recomendação geral desta medida que, por outro lado, 
poderia ser utilizada como um tratamento suplementar na hipertensão ${ }^{11}$.

Tem sido demonstrado que aumentos na ingestão de potássio que levam a aumentos no nível de potássio plasmático, inversamente se associam à diminuição da pressão sangüínea e à diminuição da mortalidade por acidente vascular cerebral e por doenças cardíacas ${ }^{12,13}$. Para explicar este benefício, vários mecanismos têm sido sugeridos. Estes incluem redução da atividade adrenérgica neural ${ }^{14}$, diminuição dos níveis da renina plasmática ${ }^{14}$, inibição da formação de radicais livres ${ }^{15}$ e aumento da atividade da enzima $\mathrm{Na}^{+} / \mathrm{K}^{+}$-ATPase, aumentando a captação celular do potássio e reduzindo o sódio intracelular ${ }^{16}$.

A suplementação dietética de potássio pode ser realizada a partir de uma dieta rica em alimentos com alto teor de potássio, da utilização de sal de cozinha contendo cloreto de potássio, ou ainda de preparações de potássio disponíveis no mercado. Em casos de depleção de cloreto associada à depleção de potássio, como pode ocorrer durante o uso de diuréticos, a reposição de cloreto de potássio seria mais adequada ${ }^{16}$. Lotaif et al. ${ }^{17}$ demonstraram significante redução da pressão arterial em pacientes com hipertensão arterial leve, sem o uso de medicação anti-hipertensiva, com a utilização de um sal de cozinha contendo $50 \%$ de $\mathrm{NaCL}$ e $50 \%$ de $\mathrm{KCL}$.

A suplementação via cloreto de potássio no sal de cozinha traria vantagens adicionais, se considerarmos que as preparações de cloreto de potássio podem causar intolerância gástrica. A desvantagem, no entanto, seria de não poder determinar a quantidade a ser ingerida.

Neste contexto, o exercício físico previne ou melhora as condições associadas a hiperinsulinemia, incluindo dislipidemia, hipertensão e aterosclerose em homens ${ }^{18}$. Aumenta a sensibilidade à insulina, independente da redução de peso, provavelmente pelo aumento dos receptores de insulina ${ }^{19,20}$, e por aumento da atividade síntese de glicogênio ${ }^{21}$.
Considerando-se que as mudanças no estilo de vida podem auxiliar no tratamento de hipertensão arterial22, neste estudo objetivamos avaliar a eficácia simultânea da suplementação de potássio, a partir de um sal de cozinha contendo cloreto de potássio, da redução de peso por meio de dieta hipocalórica e do aumento da atividade física por meio da caminhada (40 minutos, 3 vezes por semana), como medidas auxiliares para promover redução da pressão arterial e do grau de resistência à insulina em pacientes hipertensos, com excesso de peso e em monoterapia com diuréticos.

\section{CASUÍSTICA E MÉTODOS}

Foram incluídos neste estudo 28 pacientes hipertensos e obesos ou com sobrepeso, com idade entre 20 e 70 anos, monitorados no Ambulatório de Hipertensão e Diabetes do Hospital do Rim e Hipertensão da Universidade Federal de São Paulo. O estudo prospectivo randomizado, duplo-cego, com 12 semanas de duração, foi aprovado pelo Comitê de Ética em Pesquisa da Universidade Federal de São Paulo, e todos os pacientes assinaram o termo de consentimento após terem sido informados sobre o estudo. Os pacientes foram alocados aleatoriamente em dois grupos: um grupo controle $(n=13)$, que utilizou o sal de cozinha comum com $100 \%$ de cloreto de sódio grupo sal normal (SA), e o grupo experimental $(n=15)$, com o sal de cozinha contendo $50 \%$ de cloreto de sódio e $50 \%$ de cloreto de potássio grupo sal de potássio (SB). Ambos os produtos foram acondicionados em embalagens semelhantes de 1k. Para inclusão dos pacientes no estudo, foram considerados os seguintes critérios: uso exclusivo de diuréticos (hidroclorotiazida ou clortaridona) por no mínimo três semanas; níveis de pressão arterial sistólica $>140 \mathrm{mmHg}$ e $<160 \mathrm{mmHg}$ e de pressão arterial diastólica $>90 \mathrm{mmHg}$ e $<105 \mathrm{mmHg}$; obesidade ou sobrepeso com índice de massa corporal (IMC) $\geq 27 \mathrm{~kg} / \mathrm{m}^{2}$, medidos durante a consulta médica; e, afinal, distribuição central da gordura corporal 
caracterizada por uma relação entre as circunferências da cintura e do quadril (RCQ) $\geq 0,85$ para mulheres e $\geq 0,95$ para homens. Foram excluídos os pacientes com evidências clínicas ou laboratoriais de cardiopatias, acidentes vasculares prévios, discrasias sangüíneas, doença renal, hepatopatias, diabetes mellitus, níveis séricos de potássio $\geq 5,0$ ou $<3,0 \mathrm{mEq} / \mathrm{L}$, ou em uso de outros anti-hipertensivos que não os diuréticos.

Nas 12 semanas de tratamento, os pacientes foram avaliados em cinco ocasiões. Em todas as visitas foram observados os parâmetros de pressão arterial, freqüência cardíaca, peso corporal com cálculo do índice de massa corporal, e medidas das circunferências da cintura (CC) e do quadril (CQ). Os valores da pressão arterial sistólica e diastólica durante a consulta médica foram determinados pelo método auscultatório. As medidas foram realizadas com o manguito colocado cobrindo os dois terços na parte superior do braço com o paciente sentado, após 5 minutos em repouso, sendo registrados os valores da pressão sistólica e diastólica, que correspondem às fases I e $V$ dos sons de Korotkoff, respectivamente.

Todos os pacientes receberam orientação dietética individualizada, com base no registro alimentar fornecido por cada um deles, sendo que a estimativa da taxa metabólica basal, em quilocalorias (kcal), foi baseada na avaliação da composição corporal. O regime dietético foi normal em nutrientes e atendeu às necessidades nutricionais diárias dos pacientes (taxa metabólica, mais fator atividade), mas projetou-se um déficit de $1000 \mathrm{kcal} / \mathrm{dia}$, com o objetivo de promover perda ponderal, prescrevendo-se no entanto, um mínimo de $1200 \mathrm{kcal}$. Os pacientes foram estimulados à realização de atividade física (caminhada de 40 minutos, 3 vezes por semana).

Para testar a aderência dos pacientes ao consumo do sal, em todas as visitas foram realizadas coletas de urina durante 12 horas noturnas para medir o sódio, o potássio e a creatinina. Para corrigir as eventuais perdas de urina no momento da coleta e fazer uma estimativa da excreção diária, os valores da excreção urinária de sódio e potássio foram divididos pela quantidade de creatinina excretada na mesma amostra de urina e expressos em $\mathrm{mEq} / \mathrm{g}$. Na primeira e na última visita de acompanhamento, foram realizados testes orais de tolerância à glicose, com determinação da insulina sérica e da glicemia no jejum, da glicemia aos 30, 60, 90 e 120 minutos após sobrecarga oral de $75 \mathrm{~g}$ de glicose, além da determinação dos níveis séricos de sódio, potássio, creatinina, colesterol total e suas frações e triglicérides, também aos 120 minutos após a sobrecarga de glicose. A monitorização ambulatorial da pressão arterial (MAPA) foi feita por meio do método oscilométrico, com utilização de aparelho de medição indireta, SPACE LABS-90207; as medidas da composição corporal, foram obtidas pelo método de bioimpedância, utilizando-se o aparelho modelo Quantum-BIA 101 Q, RJL Systems.

Para avaliar a resistência à insulina, foram utilizados dois índices: o HOMA [ $r=$ insulinemia no jejum (mU/L) X glicemia no jejum $(\mathrm{mmol} / \mathrm{L})] / 22,5]^{23}$, e o índice de resistência à insulina, obtido com os valores da glicemia e insulinemia avaliados 120 minutos após a sobrecarga de glicose $[|\mathrm{R}|=$ glicemia $(\mathrm{mg} / \mathrm{L}) X$ insulina plasmática $\left./ 10^{4}\right]^{24}$. As áreas sob as curvas da glicose foram calculadas utilizando-se a Fórmula de Vechio: área $=A / 2+B+C+D+E / 2$, sendo que $A, B, C, D$ e E correspondem aos valores da glicemia no jejum e aos 30, 60, 90 e 120 minutos após a sobrecarga de glicose.

Para análise estatística dos resultados obtidos, utilizou-se o programa Jandel Sigma Stat version 2.0 - 1995. O teste " $\mathrm{t}$ " de Student para amostras independentes foi utilizado para comparações entre grupos com distribuição normal. Utilizou-se o Teste de Mann-Whitney para comparação de variáveis não paramétricas entre os grupos. Análise de variância, para mesmos indivíduos, ou análise de variância de Friedman, foi utilizada para comparação dos valores obtidos dentro de um mesmo grupo durante o tratamento. 
O teste " $\mathrm{t}$ " de Student e o teste de Wilcoxon foram utilizados para comparar valores das variáveis paramétricas e não-paramétricas respectivamente, obtidos dos dois grupos antes e ao final do tratamento. O coeficiente de correlação de Spearman $\left(r_{s}\right)$ foi calculado para testar as correlações que envolviam variáveis não-paramétricas, e para testar as correlações entre as variações nos diversos parâmetros estudados, ocorridas durante o período de observação.

\section{RESULTADOS}

Dos 28 pacientes (24 mulheres e 4 homens) incluídos neste estudo, 6 foram excluídos, 4 por necessidade de alteração da medicação anti-hipertensiva, e 2 por falta de aderência ao tratamento. Assim, 10 pacientes do grupo SA (cloreto de sódio) e 12 do grupo SB (50\% de cloreto de sódio e $50 \%$ de cloreto de potássio) concluíram o estudo. A idade dos pacientes variou de 23 a 67 anos, com média de 47,5 $\pm 11,2$ anos. Não houve escolha do sexo dos pacientes: quanto a isso, a amostra foi casual. Dos 10 pacientes do grupo SA, 7 utilizaram Clortaridona $25 \mathrm{mg}$ e 3 utilizaram Hidroclorotiazida $25 \mathrm{mg}$. Dos 12 pacientes do grupo SB, 8 utilizaram Clortaridona $25 \mathrm{mg}$ e 4 usaram Hidroclorotiazida $25 \mathrm{mg}$.

Os grupos SA e SB não apresentaram diferenças estatisticamente significativas com relação às características clínicas no início do tratamento, exceção feita à relação entre as medidas da cintura e do quadril (RCQ) $(p=0,017)$ (Tabela 1).

Durante o estudo, os pacientes dos dois grupos apresentaram perda de peso significativa de $71,8 \pm 7,6$ para $69,3 \pm 8,3 \mathrm{~kg}$ no grupo $S A$ $(p=0,020)$, e de $82,9 \pm 11,7$ para $79,4 \pm 12,7$ no grupo SB $(p=0,003)$. Esta redução de peso em termos percentuais, de $-3,5 \pm 2,0 \%$ no grupo $S A$ e de $-2,7 \pm 3,2 \%$ no grupo $S B$, não foram diferentes entre si. Apesar da redução de peso, as medidas da circunferência da cintura, nos dois grupos, não mostraram reduções significativas durante o período de observação (Tabela 2).

Com relação à composição corporal dos pacientes do grupo SB (Tabela 2), observamos que não houve variação da quantidade de água e massa magra, mas sim uma perda de massa de gordura significativa (de 29,2 $\pm 8,6$ para $26,1 \pm 9,2 \mathrm{~kg} ; p=0,004)$, o que resultou em termos percentuais no aumento da massa magra corporal de $65 \%$ para $69 \%$. Nos pacientes do grupo SA, por outro lado, a redução de peso se deveu em parte à redução da água corporal e em parte à perda de massa gorda, sendo que as variações observadas nestes dois parâmetros, analisados separadamente, não se mostraram estatisticamente significativas.

Como podemos observar na Tabela 2, os valores da relação entre as excreções urinárias basais de potássio e sódio (K/P), não foram

Tabela 1. Características dos pacientes com hipertensão, dos grupos sal normal e sal de potássio no período basal.

\begin{tabular}{|c|c|c|c|c|}
\hline \multirow{2}{*}{ Características } & \multicolumn{2}{|c|}{ Sal normal $(n=10)$} & \multicolumn{2}{|c|}{ Sal de potássio $(n=12)$} \\
\hline & M & $\mathrm{DP}$ & M & DP \\
\hline Raça & \multicolumn{2}{|c|}{5 branca/4 parda } & \multicolumn{2}{|c|}{5 branca/4 negra } \\
\hline Idade & $50,10 \pm$ & 8,10 & $45,40 \pm$ & $\pm 13,20$ \\
\hline IMC (kg/m2) & $30,20 \pm$ & 2,70 & $32,50 \pm$ & $\pm 13,20$ \\
\hline $\mathrm{RCQ}$ & $0,98 \pm$ & 0,07 & $0,92 \pm$ & $\pm 0,04^{*}$ \\
\hline PAS (mmHg) & $139,60 \pm$ & 11,90 & $136,20 \pm$ & 6,00 \\
\hline PAD (mmHg) & $92,30 \pm$ & 5,10 & $93,80 \pm$ & 4,30 \\
\hline
\end{tabular}

* $p=0,017$ em relação ao grupo sal normal; Os valores foram obtidos na semana 0 do estudo (período basal); IMC= índice de massa corporal; $\mathrm{RCQ}=$ razão cintura quadril; PAS= pressão arterial sistólica; $\mathrm{PAD}=$ pressão arterial diastólica de consultório. 
estatisticamente diferentes nos grupos SA e SB no início do estudo. Após o tratamento, esta relação no grupo SB apresentou um aumento estatisticamente significativo de 0,30 $\pm 0,27$ para $0,55 \pm 0,29 ; p=0,045$, refletindo a maior ingestão de potássio destes pacientes, enquanto no grupo SA esta relação não se alterou. Apesar deste aumento apresentado no grupo SB, apenas 29\% do cloreto de sódio ingerido no início, estaria sendo substituído por cloreto de potássio. A ingestão média inicial de cloreto de sódio neste grupo foi de 6,5g (70\% da excreção de sódio de 159,9mEq/g de creatinina). Se considerarmos a redução média na excreção de sódio de $30 \mathrm{mEq} / \mathrm{g}$ de creatinina ( $0,69 \mathrm{~g}$ de sódio e a $1,8 \mathrm{~g}$ de cloreto de sódio), e o aumento de $23,4 \mathrm{mEq}$ na excreção de potássio $(0,91 \mathrm{~g}$ de potássio e a $1,74 \mathrm{~g}$ de cloreto de potássio), podemos considerar que aproximadamente $1,8 \mathrm{~g}$ de cloreto de potássio substituiu $1,8 \mathrm{~g}$ de cloreto de sódio (29\%).

A quantidade administrada de sal de cozinha contendo cloreto de potássio não impediu a redução dos níveis séricos de potássio abaixo do limite inferior de normalidade de $3,8 \mathrm{mEq} / \mathrm{L}$, em 5 dos 12 pacientes tratados com o sal contendo cloreto de potássio. De forma semelhante, 4 dos
10 pacientes do grupo que recebeu somente cloreto de sódio reduziram os níveis de potássio a valores inferiores a $3,8 \mathrm{mEq} / \mathrm{L}$. Observou-se que os pacientes que já estavam fazendo uso do diurético por longos períodos $(n=11)$, tenderam a apresentar menor redução dos níveis séricos de potássio durante o período de estudo, do que aqueles $(n=11)$ que iniciaram o seu uso três semanas antes da divisão aleatória dos grupos $(-0,06 \pm 0,31$ vs $0,32 \pm 0,35 \mathrm{mEq} / \mathrm{L} ; p=0,09)$, independentemente do sal utilizado.

As medidas da pressão arterial durante a consulta do grupo SB revelaram redução da pressão arterial sistólica e diastólica no final do tratamento, em relação aos valores da semana inicial $(p<0,001)$. Já a pressão arterial medida durante a consulta do grupo SA não apresentou redução no final do tratamento (Tabela 3).

Para as medidas realizadas pela monitorização ambulatorial da pressão arterial das 24 horas (MAPA), conforme a Tabela 3, no período basal, as médias diurnas e noturnas das pressões sistólica e diastólica, entre os grupos SA e SB não se mostraram diferentes. Após o tratamento, no grupo SB, observamos que a média diurna da PAS se reduziu, embora as variações não tenham

Tabela 2. Medidas antropométricas, composição corporal e excreção urinária de sódio e potássio, nos períodos basal e final do tratamento dos grupos sal normal e sal de potássio.

\begin{tabular}{|c|c|c|c|c|c|c|c|c|c|c|}
\hline \multirow{3}{*}{ Medidas } & \multicolumn{5}{|c|}{ Sal normal } & \multicolumn{5}{|c|}{ Sal de potássio } \\
\hline & \multicolumn{3}{|c|}{ Basal } & \multicolumn{2}{|r|}{ Final } & \multicolumn{3}{|c|}{ Basal } & \multicolumn{2}{|r|}{ Final } \\
\hline & $M$ & & DP & $\mathrm{M}$ & DP & M & & DP & $\mathrm{M}$ & DP \\
\hline IMC $\left(\mathrm{kg} / \mathrm{m}^{2}\right)$ & 30,20 & \pm & 2,70 & 29,20 & $\pm 3,00$ * & 32,50 & \pm & 3,60 & 30,90 & $\pm 3,90 * *$ \\
\hline $\mathrm{CC}(\mathrm{cm})$ & 101,10 & \pm & 9,60 & 99,60 & $\pm 10,80$ & 100,70 & \pm & 9,10 & 97,80 & $\pm 11,20$ \\
\hline \multicolumn{11}{|c|}{ Composição Corporal } \\
\hline Água (kg) & 35,50 & \pm & 4,20 & 34,70 & 4,60 & 39,10 & \pm & 5,60 & 38,90 & 5,40 \\
\hline MM (kg) & 48,50 & \pm & 5,90 & 47,50 & 6,30 & 53,50 & \pm & 7,40 & 53,20 & 7,40 \\
\hline MG (kg) & 23,60 & \pm & 5,40 & 22,60 & 7,50 & 29,20 & \pm & 8,60 & 26,10 & $9,20 *$ \\
\hline \multicolumn{11}{|l|}{ Urina (U) } \\
\hline $\mathrm{K}_{\mathrm{u}}(\mathrm{mEq} / \mathrm{g})$ & 48,20 & \pm & 22,50 & 40,90 & $\pm 17,20$ & 38,80 & \pm & 18,60 & 62,30 & $\pm 29,70^{*}$ \\
\hline $\mathrm{Na}_{\mathrm{u}}(\mathrm{mEq} / \mathrm{g})$ & 171,50 & \pm & 59,30 & 177,90 & $\pm 99,50$ & 159,90 & \pm & 62,40 & 130,00 & $\pm 50,20$ \\
\hline $\mathrm{K}_{\mathrm{u}} / \mathrm{Na}_{\mathrm{u}}$ & 0,29 & \pm & 0,12 & 0,31 & $\pm \quad 0,24$ & 0,30 & \pm & 0,27 & 0,55 & $\pm 0,29 *$ \\
\hline
\end{tabular}

${ }^{*} p<0,05$ vs basal; ${ }^{* *} p<0,001$ vs basal. IMC= índice de massa corporal; $C C=$ circunferência da cintura; $M M=$ massa magra; $M G=$ massa gorda; $\mathrm{K}=$ potássio; $\mathrm{Na}=$ sódio. 
atingido níveis de significância estatística. Já no grupo SB, as médias noturnas de pressão arterial sistólica e diastólica apresentaram redução significativa. No grupo SA observamos uma diferença significativa nas médias diurnas da PAS e de PAD, mas nenhuma diferença nas médias noturnas.

Considerando-se a pressão arterial de 24 horas, a pressão sistólica apresentou redução significativa nos dois grupos, SA e SB, e a pressão arterial diastólica apresentou redução significativa somente no grupo SB. Entretanto, as variações observadas não mostraram diferenças estatisticamente significativas entre os dois grupos. Analisando os dados de todos os pacientes em conjunto, observamos uma redução de 4,9 \pm $6,9 \mathrm{mmHg}$ na média da pressão sistólica e redução de $8,4 \pm 9,4 \mathrm{mmHg}$ na média da pressão diastólica do período de 24 horas.

Nos dois grupos, considerando os períodos basal e final, não foram observadas alterações significativas no perfil lipídico do plasma (Tabela 4). Em nenhum dos grupos houve alterações significativas na área sob a curva glicêmica, obtida após a sobrecarga de glicose (Figura 1). No grupo SA, houve aumento da glicemia na verificação aos 60 minutos, de $158,50 \pm 47,26$ para $184,70 \pm 52,07 ; p=0,040 ;$ no entanto, este aumento não resultou em alteração significativa na área sob a curva glicêmica.

Com relação à resistência à insulina, os valores da mediana e intervalo de confiança dos índices HOMA não mostraram alterações significativas, tanto no grupo SA, de 3,2 $\left(2,1 \_4,9\right)$ para 2,8 $\left(1,9 \_6,3\right)$, como no grupo SB, de 3,5 $\left(2,4 \_7,0\right)$ para 3,7(2,3_6,5). O IRI se elevou de $0,88\left(0,6 \_2,9\right)$ para $1,4\left(0,5 \_1,9\right)$ no grupo SA e se reduziu de $1,0\left(0,5 \_3,5\right)$ para $0,72\left(0,4 \_1,8\right)$ no grupo SB (Tabela 4), sem que estas alterações se mostrassem significativas do ponto de vista estatístico.

Uma vez que os dois grupos se mostraram muito semelhantes quanto às variações observadas nos diversos parâmetros estudados, avaliamos o impacto da redução ponderal sobre a pressão arterial em todos os pacientes analisados em conjunto. Verificamos que as reduções percentuais nos valores da média da pressão arterial sistólica no período de 24 horas mostraram correlação significativa com as reduções percentuais observadas na medida da circunferência da cintura $\left(r_{s}=0,624 ; p=0,002\right)$, mas não com as reduções percentuais do IMC $\left(r_{s}=-0,1 ; p=0,653\right)$ ou da massa gorda $\left(r_{s}=0,04\right.$; $p=0,850$ ).

Embora as variações nos índices de resistência à insulina, durante o estudo, não tenham sido estatisticamente significativas, pudemos observar que, em termos percentuais, as variações do HOMA se correlacionaram positivamente com as variações da circunferência da cintura $\left(r_{s}=0,490 ; p=0,020\right)$ e que as variações do índice IRI se correlacionaram positivamente com as variações do IMC $\left(r_{s}=0,452 ; p=0,040\right)$ e da massa gordurosa $\left(r_{s}=0,55 ; p=0,010\right)$. Por outro lado, não foram observadas correlações entre as variações nos valores da pressão arterial e nas variações ocorridas nos índices de resistência à insulina.
Antes do tratamento

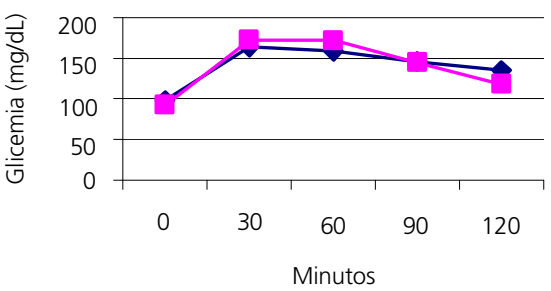

Depois do tratamento

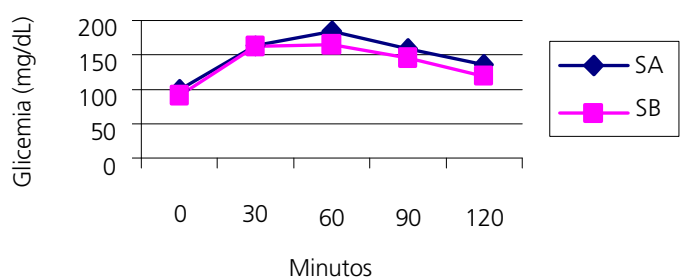

Figura 1. Média dos valores de glicemia no teste de tolerância à glicose antes e ao final do tratamento dos Grupos sal normal (SA) e de potássio (SB). 
Tabela 3. Medidas da Pressão Arterial sistólica e diastólica para os períodos diurno e noturno e de 24 horas durante a MAPA, e variação das pressões sistólica e diastólica de consultório nos períodos basal e final do tratamento nos grupo de sal normal e de potássio.

\begin{tabular}{|c|c|c|c|c|c|c|c|c|c|}
\hline \multirow{3}{*}{ Pressão Arterial } & \multicolumn{4}{|c|}{ Sal normal } & \multicolumn{5}{|c|}{ Sal de potássio } \\
\hline & \multicolumn{2}{|c|}{ Basal } & \multicolumn{2}{|r|}{ Final } & \multicolumn{2}{|c|}{ Basal } & \multicolumn{3}{|c|}{ Final } \\
\hline & M & DP & $M$ & DP & $M$ & DP & $M$ & & $\mathrm{DP}$ \\
\hline \multicolumn{10}{|l|}{ Mapa } \\
\hline Diurno/ PAS & 137,20 & $\pm 14,00$ & 130,60 & $\pm 12,30^{*}$ & 130,70 & $\pm 7,20$ & 126,30 & \pm & 6,10 \\
\hline Diurno/PAD & 86,80 & $\pm \quad 9,80$ & 82,70 & $\pm 9,00^{*}$ & 86,20 & $\pm 6,00$ & 82,70 & \pm & 4,60 \\
\hline Noturno/PAS & 126,80 & $\pm 17,60$ & 126,70 & $\pm 16,40$ & 119,40 & $\pm 8,00$ & 114,30 & \pm & $7,30 *$ \\
\hline Noturno/PAD & 76,40 & $\pm 12,20$ & 76,20 & $\pm 11,90$ & 75,90 & $\pm 4,10$ & 71,00 & \pm & $3,70 *$ \\
\hline 24 Horas/PAS & 134,70 & $\pm 14,80$ & 130,20 & $\pm 12,60$ * & 128,20 & $\pm 7,40$ & 122,90 & \pm & $5,70 *$ \\
\hline 24 Horas/PAD & 84,40 & $\pm 10,20$ & 81,40 & $\pm \quad 8,90$ & 84,00 & $\pm 5,70$ & 79,50 & \pm & $3,90 *$ \\
\hline \multicolumn{10}{|l|}{ Consultório } \\
\hline PAS & 139,60 & $\pm 11,95$ & 130,10 & $\pm 10,50$ & 136,17 & $\pm 6,01$ & 120,92 & \pm & $13,17 * *$ \\
\hline PAD & 92,30 & $\pm \quad 5,12$ & 87,40 & $\pm 10,30$ & 93,83 & $\pm 4,39$ & 82,50 & \pm & $6,67 * * *$ \\
\hline
\end{tabular}

(*) $p<0,05$ em relação ao basal; ${ }^{* *)} p=0,001$ em relação ao basal; ${ }^{(* *)} p<0,001$ em relação ao basal PAS= pressão arterial simbólica; $\mathrm{PAD}=$ pressão arterial diastática; MAPA= monitoração ambulatorial da pressão arterial.

Tabela 4. Níveis de sódio, potássio e creatinina séricos, perfil lipídico do plasma, área sob a curva da glicose, insulinemia no jejum e pós sobrecarga oral de glicose (média土DP) e índices de resistência à insulina-IRI e HOMA (mediana), nos períodos basal e final do tratamento.

\begin{tabular}{|c|c|c|c|c|c|c|c|c|c|c|}
\hline \multirow{3}{*}{ Medidas } & \multicolumn{5}{|c|}{ Sal normal } & \multicolumn{5}{|c|}{ Sal de potássio } \\
\hline & \multicolumn{3}{|c|}{ Basal } & \multicolumn{2}{|r|}{ Final } & \multicolumn{2}{|r|}{ Basal } & \multicolumn{3}{|c|}{ Final } \\
\hline & M & & DP & M & DP & M & DP & M & & DP \\
\hline $\mathrm{Na}(\mathrm{mEq} / \mathrm{L})$ & 139,90 & \pm & 2,20 & 138,70 & 2,70 & 138,80 & 2,90 & 140,00 & \pm & 2,60 \\
\hline $\mathrm{K}(\mathrm{mEq} / \mathrm{L})$ & 4,10 & \pm & 0,60 & 3,90 & 0,40 & 4,10 & 0,70 & 3,90 & \pm & 0,40 \\
\hline $\mathrm{Cr}(\mathrm{mg} / \mathrm{dL})$ & 0,91 & \pm & 0,10 & 0,95 & 0,10 & 0,92 & 0,10 & 0,94 & \pm & 0,10 \\
\hline $\mathrm{CT}(\mathrm{mg} / \mathrm{dL})$ & 212,70 & \pm & 39,90 & 211,40 & 31,40 & 205,80 & 25,10 & 197,20 & \pm & 22,20 \\
\hline $\mathrm{HDL}-\mathrm{CL}(\mathrm{mg} / \mathrm{dL})$ & 55,40 & \pm & 14,40 & 55,00 & 18,00 & 46,70 & 8,70 & 41,90 & \pm & 16,10 \\
\hline LDL-CL (mg/dL) & 130,30 & \pm & 36,50 & 129,50 & 32,40 & 132,70 & 21,70 & 114,50 & \pm & 39,50 \\
\hline $\mathrm{TG}(\mathrm{mg} / \mathrm{dL})$ & 134,50 & \pm & 84,40 & 134,00 & $\pm \quad 82,90$ & 131,70 & 68,30 & 129,80 & \pm & 71,40 \\
\hline ASCG (mg/h) & 584,10 & & 149,70 & 625,60 & $\pm 156,50$ & 593,80 & $\pm 160,40$ & 579,80 & & 38,30 \\
\hline INS J (uU/mL) & 15,40 & \pm & 7,30 & 15,90 & $\pm \quad 9,50$ & 19,10 & $\pm \quad 10,90$ & 18,90 & \pm & 13,00 \\
\hline INS $120(\mathrm{uU} / \mathrm{mL})$ & 107,90 & \pm & 57,90 & 105,90 & $\pm \quad 61,40$ & 127,30 & $\pm \quad 91,40$ & 102,80 & \pm & 96,40 \\
\hline $\mid \mathrm{RI}$ & 0,88 & \pm & $\left(0,6 \_2,9\right)$ & 1,40 & $\pm\left(0,5 \_1,9\right)$ & 1,00 & $\pm\left(0,5 \_3,5\right)$ & 0,72 & $\pm(C$ & $\left.0,4 \_1,8\right)$ \\
\hline HOMA & 3,20 & \pm & $\left(2,1 \_4,9\right)$ & 2,80 & $\pm\left(1,9 \_6,3\right)$ & 3,50 & $\pm\left(2,4 \_7,0\right)$ & 3,70 & \pm( & $\left.2,3 \_6,5\right)$ \\
\hline
\end{tabular}

$\mathrm{Na}=$ sódio; $\mathrm{K}=$ potássio; $\mathrm{Cr}=$ creatinina; $\mathrm{CT}=$ colesterol total; $\mathrm{HDL}-\mathrm{CL}=\mathrm{HDL}$-colesterol; $\mathrm{LDL}-\mathrm{CL}=\mathrm{HDL}$ colesterol; $\mathrm{TG}=$ triglicérides; $\mathrm{ASCG}=$ área sob as curvas de glicose; INS J= isulina no jejum; INS 120= insulina pós prandial; Mediana= Intervalo de confiança dos índices de resistência à insulina (RI) IRI e HOMA.

\section{I S C U S S Ã O}

Os resultados do presente estudo mostraram os benefícios no controle da pressão arterial obtidos com a redução do peso corporal, mas não confirmaram os benefícios da suplementação de potássio a partir da utilização do sal de cozinha para pacientes em uso de diuréticos, conforme sugerido em estudos anteriores. 
Por intermédio da análise das quantidades de potássio excretadas na urina, apenas cerca de $30 \%$ do cloreto de sódio consumido no início do estudo foi substituído por cloreto de potássio no grupo SB, resultando numa suplementação dietética de potássio insuficiente para impedir uma redução na média dos níveis séricos de potássio; esta redução, ela mesma não significativa do ponto de vista estatístico, não diferiu daquela observada no grupo controle. Nossos dados, portanto, diferem dos obtidos por Lotaif et al. ${ }^{17}$ que conseguiram corrigir a hipocalemia em pacientes hipertensos em uso de diuréticos com a administração do sal de cozinha contendo cloreto de potássio. No referido estudo, entretanto, a maior aderência à recomendação de substituir o sal de cozinha comum pelo sal contendo potássio foi conseguida pelo fato de terem sido incluídos apenas pacientes que faziam todas as refeições em casa, na maioria donas de casa e aposentados.

Em nosso estudo, o fato de terem sido incluídos pacientes que faziam suas refeições fora de casa, apesar da garantia de que as refeições eram todas preparadas em casa e levadas para o trabalho, pode ter dificultado o consumo do sal contendo cloreto de potássio. De qualquer forma, nossos resultados indicam que esta forma de suplementação de potássio para pacientes hipertensos em uso de diuréticos, embora possa ser eficiente para evitar hipocalemia, é pouco efetiva para os pacientes que trabalham e fazem as refeições fora de casa, pela dificuldade que encontram em seguir a recomendação. Assim, constatamos que a maior desvantagem na suplementação de potássio via sal de cozinha está no fato de não podermos determinar a quantidade a ser ingerida. Em populações como a nossa, com baixo consumo de alimentos ricos em potássio, a preocupação é com a reposição insuficiente que, em pacientes mais propensos à hipocalemia e resistentes à ação da insulina, pode resultar em intolerância à glicose, como já demonstrado anteriormente ${ }^{4,25}$.

Durante o nosso estudo, os pacientes dos dois grupos apresentaram perda de peso de forma semelhante. No período de tratamento de 12 semanas, as perdas de peso de 3,5\% no grupo com sal comum e de $2,7 \%$ no grupo consumindo sal com cloreto de potássio, foram semelhantes às de outros estudos em que os pacientes foram submetidos à dieta hipocalórica e tratados com placebo de medicamentos anti-obesidade ${ }^{26,27}$. A redução de peso observada pode justificar a redução nas médias de pressão arterial obtidas pela MAPA, nos dois grupos, como demonstrado em outros estudos ${ }^{8,28}$. As reduções nos níveis pressóricos na monitorização de 24 horas foram também muito semelhantes nos dois grupos.

Os parâmetros de resistência à insulina, assim como a área sob a curva da glicose e o perfil lipídico do plasma, não se alteraram nos dois grupos durante o estudo. Estes resultados são compatíveis com resultados anteriormente obtidos em nossos pacientes, nos quais perdas de peso inferiores a 5\% não resultaram em melhora das condições metabólicas ${ }^{26}$.

O fato de os dois grupos terem se comportado de forma muito semelhante com relação a todos os parâmetros avaliados, nos levou a analisar alguns aspectos do tratamento, considerando-se os dois grupos em conjunto, como se constituíssem um único grupo. Nosso estudo demonstrou que modificações no estilo de vida, resultando em reduções mesmo pequenas do peso corporal, podem ser úteis no sentido de auxiliar no controle da pressão arterial. Quando analisamos todos os pacientes em conjunto, observamos uma redução no índice de massa corporal que, em média, atingiu cerca de 3\% do valor basal e resultou em reduções na pressão arterial de 24 horas. Estas atingiram 4,9 $9,9 \mathrm{mmHg}$ na pressão sistólica e $8,4 \pm 9,4 \mathrm{mmHg}$ na pressão diastólica. Estas reduções não são desprezíveis, se considerarmos que todos os pacientes já se encontravam em monoterapia com diuréticos. Este tipo de intervenção pode levar os valores da pressão arterial aos níveis de controle atualmente recomendados, evitando-se a necessidade de administrar outro medicamento para melhor controle da hipertensão. Trata-se, portanto, de conseguir, por meio das mudanças no estilo de 
vida, um benefício adicional em relação àquele já obtido pelo uso da medicação anti-hipertensiva, evitando-se introdução de outros anti-hipertensivos para melhor controle pressórico. Isto é especialmente importante considerando-se que as taxas de controle da hipertensão arterial têm-se mostrado baixas em todo o mundo, como atestam os dados obtidos nos Estados Unidos, onde esta taxa é inferior a $27 \% 29$.

Nossos dados tornam-se particularmente importantes se considerarmos que a maioria dos estudos que avaliaram os efeitos de intervenções não-medicamentosas sobre a pressão arterial incluiu pacientes hipertensos que não se encontravam sob medicação anti-hipertensiva. Nossos resultados são concordantes com aqueles de outro estudo, recentemente publicado, em que mudanças no estilo de vida, avaliadas em conjunto, trouxeram também benefícios a pacientes hipertensos submetidos a monoterapia medicamentosa para hipertensão arterial ${ }^{30}$.

Embora no início deste estudo não tenhamos observado nos pacientes tratados com diuréticos uma correlação significativa entre os valores da pressão arterial e a circunferência da cintura, durante o estudo foi possível verificar que as variações ocorridas nas médias dos valores da pressão arterial nas 24 horas se correlacionaram significativamente com as variações ocorridas na circunferência da cintura, mas não com as variações no IMC. Estes resultados sugerem que, a redução da gordura visceral abdominal pode ser mais importante que a redução da massa gorda total para a redução da pressão arterial. Embora a quantidade de gordura visceral possa influenciar o grau de resistência à insulina ${ }^{26}$, a associação observada entre as reduções da pressão arterial e as reduções na circunferência da cintura se mostrou independente de variações no grau de resistência à insulina, uma vez que não foram observadas correlações entre as variações pressóricas e as variações nos índices de resistência à insulina. Por outro lado, o fato de as variações nos índices de resistência à insulina se correlacionarem positivamente com as variações na circunferência da cintura e com as variações na gordura corporal, sugere o efeito benéfico da redução de peso sobre a resistência à insulina em pacientes hipertensos com distribuição central da gordura corporal.

\section{O N C L U S Ã O}

A perda de peso e o incentivo à pratica de exercícios físicos tiveram um efeito benéfico em pacientes hipertensos com excesso de peso, em uso de medicação anti-hipertensiva, resultando na redução da pressão arterial. A suplementação de potássio a partir do sal de cozinha contendo cloreto de potássio, em pacientes hipertensos obesos em uso de diurético, se mostrou insuficiente para impedir a queda dos níveis séricos de potássio e não resultou em nenhum efeito aditivo àquele obtido pela perda de peso sobre a redução da pressão arterial e metabolismo dos carboidratos.

\section{REFERÊ NCIAS}

1. Ribeiro AB, Kohlmann Jr O, Marson O, Zanella MT, Ramos OL. Hypertension: A major public health problem in Brazil. Drugs. 1988; 35(Suppl 1):1-5.

2. The sixth report of the Joint National Committee on prevention, detection, evaluation, and treatment of high blood pressure (JNC VI). Arch Intern Med. 1997; 157(21):2413-46.

3. Tharkur V, Robert R, Reisin E. Obesity, hypertension, and the heart. Am J Med Sci. 2001; 321(4):242-8.

4. Plavnick FL, Rodrigues CIS, Zanella MT, Ribeiro AB. Hypokalemia, glucose intolerance, and hyperinsulinemia during diuretic therapy. Hypertension. 1992; 19(2 Suppl 2):II-26-9.

5. Blaustein MP, Hamlyn JM. Pathogenesis of essential hypertension. Hypertension. 1991; 18(5 Suppl 3):III-184-95.

6. Franssila-Kallunki A, Rissanen A, Ekstrand A, Ollus A, Groop L. Effects of weight loss on substrate oxidation, energy expenditure, and insulin 
sensitivity in obese individuals. Am J Clin Nutr. 1992; 55(2):356-61.

7. The Trials of Hypertension Prevention, Phase II (TOHP II) Effects of weight loss and sodium reduction intervention on blood pressure and hypertension incidence in overweight people with high-normal blood pressure. Arch Intern Med. 1997; 157(6):657-67.

8. Stevens VJ, Obarzanec KE, Look NR, Lee IM, Appel LT, Smith West $D$, et al. Long-term weight loss and changes in blood pressure: results of the trials of hypertension prevention, phase II. Ann Intern Med. 2001; 134(1):1-11.

9. He J, Whelton PK, Appel L, Charleston J, Klag M. Long-term effects of weight loss and dietary sodium reduction on incidence of hypertension. Hypertension. 2000; 35(2):544-9.

10. Luft FC, Weinberger MH. Sodium sensitivity and resistance of blood pressure: The role of the kidney and the renin-aldosterone axis. Kidney Int Suppl. 1987; 32(2):234-41.

11. Graudal AG, Galloe AM, Garred, P. Effects of sodium restrition on blood pressure, renin, aldosterone, catecholamines, cholesterol and triglycerides. JAMA. 1998; 279(17):1383-91.

12. Whelton PK. Effects of oral potassium on blood pressure. Meta-analysis of randomized control clinical trials. JAMA. 1997; 277(20):1624-32

13. Whelton PK. Potassium in preventing and treating high pressure. Sem Nephrol. 1999; 19(5):494-9.

14. Fujita T, Katsuyuki A. Hemodynamic and endocrine changes associated with potassium supplementation in sodium-loaded hypertensives. Hypertension. 1984; 6(2):184-92.

15. McCabe RD, Michael AB, Kumud S, David BY. Potassium inhibits free radicals formation. Hypertension. 1994; 24(1):77-82.

16. Gennari FJ. Hypocalemia. N Engl J Med. 1998; 339(7):451-8.

17. Lotaif LAD, Ribeiro AB. Efeito da suplementação de potássio através do sal de cozinha na hipertensão arterial primária, leve a moderada. J Bras Nefrol. 1995; 17:214-8.
18. Pratley RE, Hagberg JM, Dengel DR, Rogus EM, Muller DC, Goldberg AP. Aerobic exercise training-induced reduction in abdominal fat and glucose-stimulated insulin responses in middle-aged and older men. J Am Geriatric Soc. 2000; 48(9): 1055-61.

19. Soma VR, Koivisto VA, Deibert D, Felig P, DeFronzo RA, Increased insulin sensitivity and insulin binding to monocytes after physical training. N Engl J Med. 1979; 301(22):1200-4.

20. Pedersen O, Beeck-Nielsen H, Heding L. Increased insulin receptors after exercise in patients with insulin-dependent diabetes mellitus. N Engl J Med. 1980; 302(16):886-92.

21. Mikines KJ, Sonne B, Trinier B, Galbo H. Effects of acute exercise and detraining on insulin action in trained men. J Appl Physiol. 1989; 66(2):704-11.

22. Conlin PR, Chow D, Miller ER 3rd, Svetkey LR, Lin $\mathrm{PH}$, Harsha DW, et al. The effect of dietary patterns on blood pressure control in hypertensive patients: Results from the dietary approaches to stop hypertension (DASH) Trial. Am J Hypertens. 2000; 13(9):949-55.

23. Matthews DR, Hosker JP, Rudenski AS, Naylor BA, Treacher DF, Turner RC. Homeostasis model assessment: insulin resistence and b-cell function from fasting plasma glucose and insulin concentrations in man. Diabetologia. 1985; 28(7):412-9.

24. Grandi AM, et al. Insulin sensitivity in obese normotensive adults: influence of family history of hypertension. Int J Obes Relat Metab Disord. 1998; 22(9):910-4.

25. Zanella MT, Baptista M, Costa CH, Kohlmann NE, Kohlmann Junior O, Ribeiro AB. Comparative effects of captopril and chlorthalidone on glucose tolerance and insulin levels in essential hypertensives. Arq Bras Cardiol. 1996; 67(1): 47-52.

26. Faria AN, Ribeiro FFF, Lerário DDG, Kolmann N, Ferreira SRG, Zanella MT. Effects of sibutramine on the treatment of obesity in patients with hypertension. Arq Bras Cardiol. 2002; 78:176-80. 
27. Bakris G, Calhoun D, Egan B, Helmann C, Dolker $M$, Kingma I. Orlistat improves blood pressure control in obese subjects with treated but inadequade controlled hypertension. J Hypertens. 2002; 20(11):2257-67.

28. Wylie-Rossett J, Wassertheil-Smaller S, Blaufox MD, Davis BR, Langford HG, Oberman A, et al. Trial of antihypertensive intervention and management: greater efficacy with weight reduction than with a sodium potassium intervention. J Am Diet Assoc. 1993; 93(4):408-15.
29. Burt VL, Artler JA, Higgins M, Horan MJ, Labarthe $D$, Whelton $\mathrm{P}$, et al. Trends in the prevalence, awareness, treatment and control of hypertension in the adult US population. Hypertension. 1995; 26(1):60-9.

30. Miller R, Erlinger TP, Young DR, Jehn M, Charleston $J$, Rhodes $D$, et al. Results of diet, exercise and weight loss intervention trial (DEW_IT). Hypertension. 2002; 40(5):612-8.

Recebido para publicação em 16 de junho de 2002 e aceito em 22 de junho de 2004. 